\title{
Performance Evaluation for a Multimodal Interface of a Smart Wheelchair with a Simulation Software
}

\author{
Amberlay Ruíz Serrano, Ruben Posada Gómez, Albino Martínez Sibaja, \\ Alberto Alfonso Aguilar Lasserre, Giner Alor Hernández and Guillermo Cortes \\ Robles \\ Instituto Tecnológico de Orizaba, Orizaba, Veracruz, México \\ amberlay_21@outlook.com, pgruben@yahoo.com, albino3_mx@yahoo.com, \\ albertoaal@yahoo.com, gineralor@hotmail.com, gc_robles@hotmail.com
}

\begin{abstract}
Handling systems for power wheelchairs are very useful for users who have difficulty moving by themselves; however, most of the current models are designed exclusively for diplegic people and quadriplegics users with a specific disability. But it doesn't exist yet a wheelchair that assist it properly and efficiently the different disabilities that may present a quadriplegic due to the level of the injury. Therefore, the main contribution for this paper was a performance evaluation for a multimodal interface system of a smart wheelchair with different control methods for patients with severe spinal cord injuries (SCI) through a software simulation to make more secure the movement of the wheelchair and thereby standardize the basics of wheelchairs for quadriplegics in a more natural way according to their disabilities.
\end{abstract}

Keywords: Power Wheelchair, Tongue Control, Speech Recognition, Motor Disability, Inter-faces, Simulation Software.

\section{Introduction}

Recently studies shows that children and adults with physical disabilities such as loss of muscle control quadriplegia or paralysis, are essentially benefited because they regain their independent mobility through manual wheelchairs, electric wheel-chairs or scooters $[1,2]$. However, the current wheelchairs, such as electric wheel-chairs or manual wheelchairs represent an alternative to partially recover the ability to move by themselves. If the wheelchair user has a very limited upper body, electric wheelchair is the perfect choice for this person. However, this is not an option for quadriplegic users, who suffer paralysis of all their limbs. The proposal of this article is to focus only on quadriplegic users, because there is a problem, which often is not taken into account when a wheelchair is designed for them and the reason is: "Not all quadriplegics are equal." On one side, an individual may need assistance all the time and on 
the other, a per-son can easily be able to live independently with appropriate assistance technology. Nowadays there is a tendency to create Human Computer Interactions (HCI) taking advantage of the skills that users with disabilities still preserve, for example, for patients who cannot use any of its members, but which can still use the movement of the eyes [3], face [4], hands [5], eyebrows [6] or voice [7]. This allows to create different control methods and apply them to a wheelchair, however each of these methods have efficiency problems and it doesn't exist smart wheelchairs developed and commercially available under these technologies in our country. The objective is to make a smart wheelchair which can enabling users with quadriplegia, retrieving a way to move themselves at the same time that this adapts to the user, regardless of whether the person has one or more disabilities. The proposed system is looking for an HCI with a multimodal interface by applying it to a smart wheelchair, to goal that different users with quadriplegia can use it, independently of the degree of SCI. To achieve this, it was also performed a study with different patients. These used the proposed system through a simulation soft-ware to learn how to use the smart wheelchair with an obstacle course in the software. After the users complete the course in a real smart wheelchair and this was compared against the results of similar systems by other researchers, to establish standards aimed at any quadriplegic people and providing a natural language for controlling the wheelchair.

\section{Multimodal Interface System}

The need to create interfaces that allow a more natural interaction with a wheelchair has motivated this system so the proposed multimodal interface integrate four control methods: a magnetic control system using a magnet, a voice control interface using a microphone, a control pad and a joystick that it can perform a simple command control with the basic moves to drive the wheelchair.

\subsection{Tongue Movement Interface}

Quadriplegics people have very limited options to care for themselves, so it is necessary to use specific skills of patients through signal pattern recognition as the movement of the tongue [8]. It is known that the tongue training with a simple protrusion task induces neural plasticity [9]. Among the different proposals to use the tongue as a method of controlling devices for assistance, most systems have direct contact with the patient [10,11]. For proposed assistance system to the wheelchair, the main goal was to use simple commands to move seamlessly through an obstacle course with precision using an interface reliable, inexpensive, discreet, minimally invasive, and easy to use. So the magnet is not placed on the tongue, but is in a dental retainer with a small rail behind the teeth, where the magnet can be moved in 5 positions by the tongue as seen in Figure 1. 
Performance Evaluation for a Multimodal Interface of a Smart Wheelchair with a Simulation Software

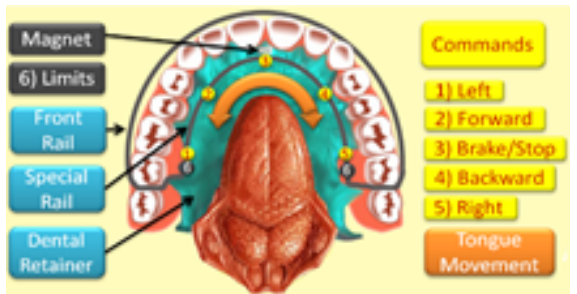

Fig. 1. Dental retainer with a magnet.

\subsection{Voice Interface}

In particular, the speech recognition system (SCS) consists of two parts: the first part is a section with a small vocabulary training that builds a model and the second is a speech recognition section which uses this model. The magnetoresistive sensor modules and microphone are placed in a headset, as seen in Figure 2, through a pair of flexible tubes which allow placing the sensors in different positions and after receiving the command signals, they are sent to the microcontroller system.

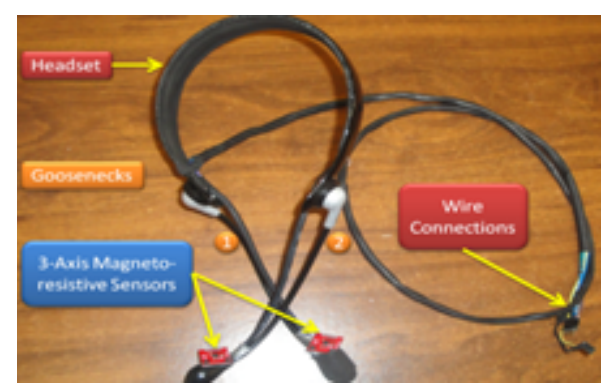

Fig. 2. Headset for Speech and Magnetic Control System

The voice commands that were used to control the system voice which can be seen in the following Table 1.

Table 1. Voice Commands for the Speech Control System

\begin{tabular}{|c|c|c|c|}
\hline Commands & Action & Commands & Action \\
\hline \hline One & Power on the system & Right & Turn to the right \\
\hline Forward & Move forward & Down & Brake motors \\
\hline Backward & Move back & Up & Shut down (Stop also engines) \\
\hline Left & Turn to the left & & \\
\hline
\end{tabular}




\subsection{Control Pad Interface and Joystick Interface}

It was used a button pad with 9 buttons, these has been programmed with directions and speeds for the wheelchair. Of course it was included a joystick because of being the most typical interface for power wheelchairs. There were included typical inter-faces that some quadriplegics can use according to their injuries. On the other hand, pad button and joystick's circuits were added to have different ways for wheel-chair's driving.

\section{Simulation Software}

Before testing the multimodal interface in the wheelchair, a computer simulation software was developed using the same features as weight and speed of the actual chair to create a small game and teach users how to properly use it intuitively. There-fore a graphical user interface (software) was performed using the computer for multimodal interface (hardware) and thereby simulate the movement of the wheelchair. The programming environment that was used for the graphical interface was "Processing", which is an open programming language resource (open source) for those who want to create animations and interactions. Moreover multimodal interface has been developed in Arduino, which was created based on Processing, so the programming is similar and the interaction between both environments is very stable. In Figure 3 is seen the GUI looks like, while in Figure 4, there is a flow diagram of the internal workings.

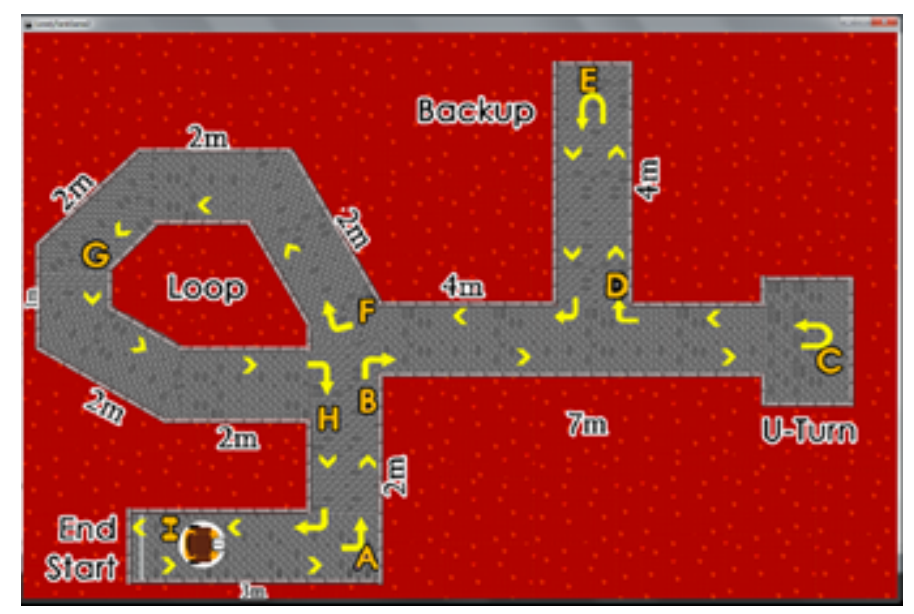

Fig. 3. GUI Software Simulation for Multimodal Interface. Track adapted for testing wheelchair performance [12,13] 


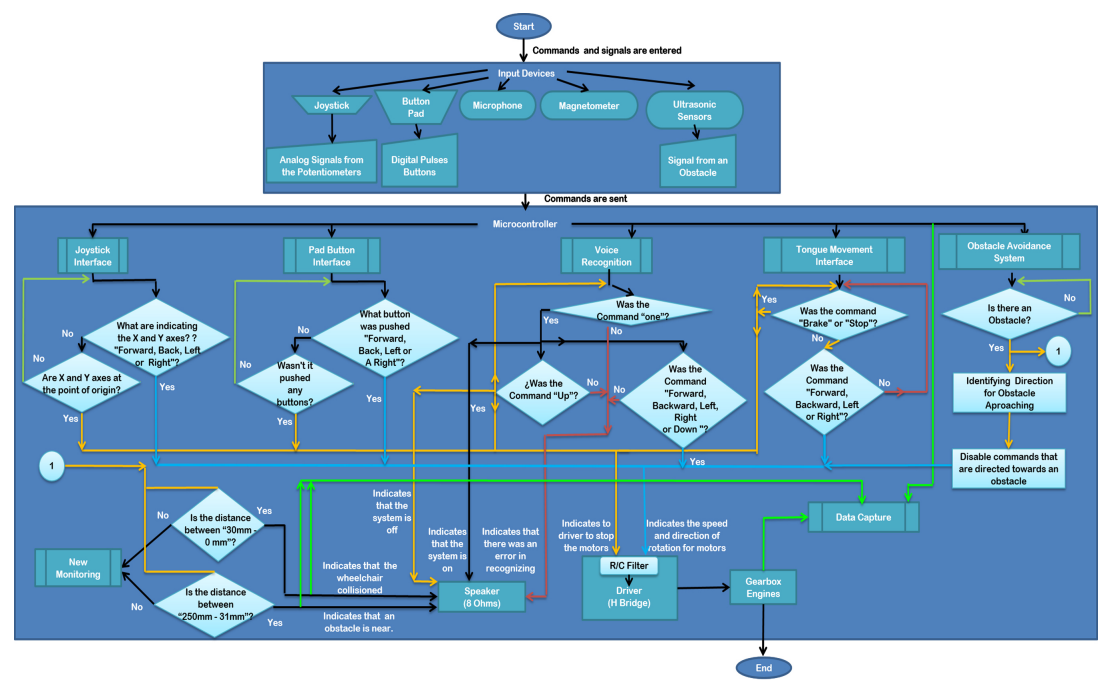

Fig. 4. Software Simulation Flow Diagram for Multimodal Interface

Flowchart shows how simulation software works. Among its main components, the input devices provide data for controlling the direction of the virtual small wheelchair. This was important, because this software is focused to achieve maximum accuracy possible to receive orders to move the wheelchair.

\subsection{Performance Tests for Simulation Software}

As can be seen, a small wheelchair and an obstacle course are shown with different signs to take appropriate route. The goal is to travel around the circuit and back to the starting point. Similarly through the usb port on the computer and serial port functions, control methods were adapted to move the wheelchair as shown in Figure 5.

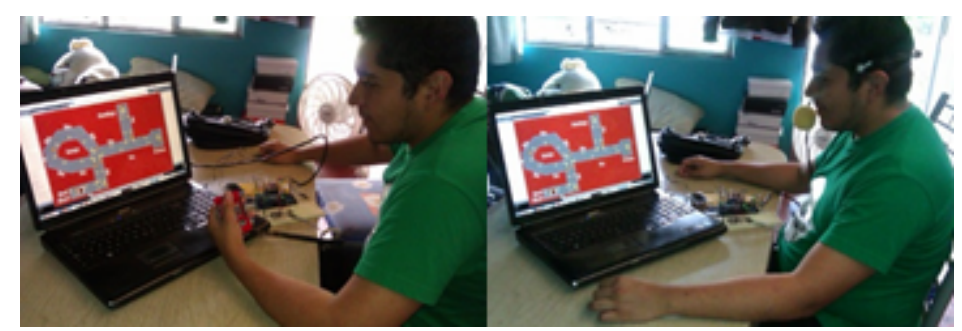

Fig. 5. GUI Software Simulation using Control Methods 
Amberlay Ruíz Serrano, Ruben Posada Gómez, Albino Martínez Sibaja, et al.

Once it worked the GUI along with the multimodal interface, it proceeded to testing with people with a physical disability in the Integral Rehabilitation Center of Orizaba (CRIO). The tests consisted of 2 people assigned to each of the control methods (joystick, button pad, voice recognition and tongue movement). In total 8 different people used the multimodal interface through the GUI. Each user had to complete the obstacle course of the GUI according to the control method which they were assigned, in addition the data for each user was stored, taking particular importance in the type of injury or disability suffered. According to international standards established by the American Spinal Injury Association (ASIA), can be classified in Full Spinal Cord Injury (FSCI) and Incomplete Spinal Cord Injury (ISCI) and in 5 types from A to E. Were also obtained data from different tests that can be seen in Table 2 as the time in which they completed the obstacle course or the times they repeated the circuit. Voice and Tongue Interface just work with a slow speed to keep safer the user.

Table 2. Performance Tests using GUI software simulation

\begin{tabular}{|c|c|c|c|c|c|}
\hline User & Injury Class & Control Method & Speed & Avg t/Lap & Avg Col/Lap \\
\hline \hline 1 & ISCI. Type E. & Pad button & Slow/Fast $69 \mathrm{~s} / 58 \mathrm{~s}$ & $6.66 / 8.56$ \\
\hline 2 & ISCI. Type E. & Pad button & Slow/Fast $74 \mathrm{~s} / 67 \mathrm{~s}$ & $5.1 / 7.53$ \\
\hline 3 & ISCI. Type C. & Joystick & Slow/Fast & $80 \mathrm{~s} / 65 \mathrm{~s}$ & $3.38 / 6.73$ \\
\hline 4 & ISCI. Type C. & Joystick & Slow/Fast $72 \mathrm{~s} / 63 \mathrm{~s}$ & $4.08 / 7.15$ \\
\hline 5 & ISCI. Type D. & Voice interface & Slow & $83 \mathrm{~s}$ & 32.21 \\
\hline 6 & ISCI. Type C. & Voice interface & Slow & $90 \mathrm{~s}$ & 28.37 \\
\hline 7 & ISCI. Type B. & Tongue interface & Slow & $53 \mathrm{~s}$ & 23.19 \\
\hline 8 & ISCI. Type B. & Tongue interface & Slow & $58 \mathrm{~s}$ & 24.53 \\
\hline
\end{tabular}

\subsection{Performance Tests in a Real Environment}

To evaluate the different ways to handle the wheelchair, the same obstacle course was used in a real environment. This time when the users handled the wheelchair seen in Figure 6. They were more cautious, so they took longer to complete the laps. However, the number of collisions was significantly reduced among drivers as shown in Table 3. Other features of this performance test were the time response taken, the average time for a full stop after selecting slow / stop and the minimum step of the wheelchair in Table 4. It should be mentioned that were used ultrasonic sensors to prevent accidents and stop the wheelchair if this was directed against an obstacle by mistake. Also whenever the sensors are activated, these count as a collision, moreover were also used a Speaker which beeps with a certain proximity to avoid a collision. Despite this sound if the wheelchair is directed against an obstacle, a different sound is activated to indicate that there was a collision. 
Performance Evaluation for a Multimodal Interface of a Smart Wheelchair with a Simulation Software

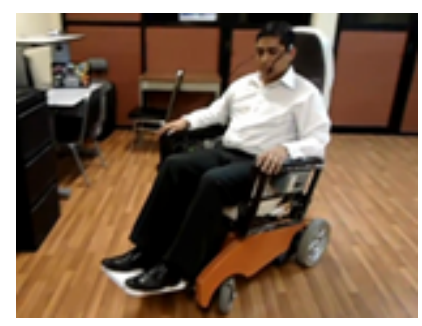

Fig. 6. Smart Wheelchair using the Multimodal Interface

Table 3. Performance Tests using Multimodal Interface apply to the Smart Wheelchair

\begin{tabular}{|c|c|c|c|c|c|}
\hline User & Injury Class & Control Method & Speed & Avg t/lap & Avg Col/lap \\
\hline \hline 1 & ISCI. Type E. & Pad button & Slow/Fast & $78 \mathrm{~s} / 67 \mathrm{~s}$ & $0.97 / 1.53$ \\
\hline 2 & ISCI. Type E. & Pad button & Slow/Fast & $92 \mathrm{~s} / 84 \mathrm{~s}$ & $0.86 / 1.29$ \\
\hline 3 & ISCI. Type C. & Joystick & Slow/Fast & $98 \mathrm{~s} / 76 \mathrm{~s}$ & $0.42 / 0.88$ \\
\hline 4 & ISCI. Type C. & Joystick & Slow/Fast & $89 / 69 \mathrm{~s}$ & $0.51 / 1.07$ \\
\hline 5 & ISCI. Type D. & Voice interface & Slow & $95 \mathrm{~s}$ & 7.37 \\
\hline 6 & ISCI. Type C. & Voice interface & Slow & $106 \mathrm{~s}$ & 9.74 \\
\hline 7 & ISCI. Type B. & Tongue interface & Slow & $72 \mathrm{~s}$ & 5.22 \\
\hline 8 & ISCI. Type B. & Tongue interface & Slow & $76 \mathrm{~s}$ & 3.28 \\
\hline
\end{tabular}

Table 4. General Characteristics of Speed and Distance of the Smart Wheelchair

\begin{tabular}{|c|c|c|c|c|}
\hline Control Method & Speed & Resp Time & Avs t to slow & Avg d/s \\
\hline \hline Pad Button & Slow fast & $0.82 \mathrm{~s} / 0.80 \mathrm{~s}$ & $0.75 \mathrm{~s} / 0.93 \mathrm{~s}$ & $0.274 \mathrm{~m} / \mathrm{s} / 0.456 \mathrm{~m} / \mathrm{s}$ \\
\hline Joystick & Slow fast & $0.83 \mathrm{~s} / 0.82 \mathrm{~s}$ & $0.73 / 0.95 \mathrm{~s}$ & $0.305 \mathrm{~m} / \mathrm{s} / 0.540 \mathrm{~m} / \mathrm{s}$ \\
\hline Voice Interface & Slow & $0.81 \mathrm{~s}$ & $0.74 \mathrm{~s}$ & $0.203 \mathrm{~m} / \mathrm{s}$ \\
\hline Tongue Interface & Slow & $0.82 \mathrm{~s}$ & $0.76 \mathrm{~s}$ & $0.236 \mathrm{~m} / \mathrm{s}$ \\
\hline
\end{tabular}

\section{Results}

The main contribution for this paper were the results of making a wheelchair with a multimodal interface. Specifically magnetic control and voice recognition was develop at low cost. These results will be discussed below.

\subsection{Control using Tongue Movements}

The control of this interface proved to be able to react to commands programmed according to the position of the magnet moving it with the tongue, also managed 
to perform the scheduled tasks for each command. During the tests, one experiment was driving the wheelchair through an obstacle course proposed by [12] this experiment was repeated 100 times with 8 different people to find significant information to compare their work with both the software simulation, as the multimodal interface. (Table 5). These results show a significant advance in tongue interface without include others.

Table 5. Comparison of Results with other Research

\begin{tabular}{|c|c|c|c|c|}
\hline & Speeds & Sim Software & Multimodal I. & Ghovanloo T. I. \\
\hline \hline Avg Col & Low & $28.36 \mathrm{~s}$ & $4.25 \mathrm{~s}$ & $1.77 \mathrm{~s}$ \\
\hline Avg t/lap & Low & $55.5 \mathrm{~s}$ & $74 \mathrm{~s}$ & $65.5 \mathrm{~s}$ \\
\hline Commands & Low & $5 \mathrm{~s}$ & $5 \mathrm{~s}$ & $5 \mathrm{~s}$ \\
\hline Time resp & Low & $0.81 \mathrm{~s}$ & $0.81 \mathrm{~s}$ & $1 \mathrm{~s}$ \\
\hline
\end{tabular}

\subsection{Speech Recognition Control}

The use of this control showed that it is able to recognize the commands programmed and then the system performs scheduled tasks for each command, also activate the sounds that were saved in the memory of speech recognition module to verify that the command was correct. Table 6 shows the results by repeating 100 times each command, on the other hand the runtime was taken using an oscilloscope to know how long it takes the system to recognize it, and finally the percentages of assertiveness are also shown with respect the number of times the system correctly recognized each command.

\section{Discussion}

Implementing this system, was a success because circuits are small. It was possible to develop a speech control that had a successful recognition rate of $95.71 \%$, which works with any type of voice; this percentage is pretty good because the speech recognition systems usually fail to be too assertive comparing with others as Coy results [14]. However, if it is required to customize a single voice is also possible to train the module to recognize only certain tones of voice. The memory limit that owns the microcontroller and the speech recognition module, restrict and limit training commands and for this reason are planned for future works, use an external memory or a computer with enough capacity to reduce the time response between the recognition of the commands and execution of assigned tasks. Finally, the magnetic control system, proposes using the magnet in the dental retainer because other systems place the magnet on the tongue with tisular adhesive which only lasts a few hours [15], or even newer systems use magnetic piercings in patients with severe disabilities [16] making 
them suffer unnecessary pain, besides the use of piercings gradually generates chipping of the dental enamel, periodontal lesions and infection tongue numbness [17]. In the other hand with the current wheelchair, the users who used it in the experiment with proper training anyone who can use the tongue will be able to drive it, although some took longer to complete the course than others.

Table 6. Percentage of assertiveness for each command of speech recognition

\begin{tabular}{|c|c|c|}
\hline Commands & Runtime & Assertiveness in speech recognition \\
\hline \hline One & $10 \mathrm{~ms}$ & $100 \%$ \\
\hline Forward & $21 \mathrm{~ms}$ & $95 \%$ \\
\hline Backward & $20 \mathrm{~ms}$ & $100 \%$ \\
\hline Left & $12 \mathrm{~ms}$ & $90 \%$ \\
\hline Right & $16 \mathrm{~ms}$ & $92 \%$ \\
\hline Down & $13 \mathrm{~ms}$ & $97 \%$ \\
\hline Up & $11 \mathrm{~ms}$ & $96 \%$ \\
\hline
\end{tabular}

Tests conducted with the control method of the tongue, resulted in an average of 4.25 collisions, each time the track was completed, resulting satisfactory comparing it with the inductive control system proposed in [13]. Future work will include a wireless system to communicate with smart phones and android operating system. Also it will be add other interfaces as used techniques of electrooculography, electromyography, or use the computer with the help of these interfaces and those already created.

\section{References}

1. Öztürk A., Dokuztug, U.F.: Effectiveness of a Wheelchair Skills Training Programme for Community-Living Users of Manual Wheelchairs in Turkey: a Randomized Controlled Trial. Clinical Rehabilitation 25, 416-424 (2011)

2. Fliess D.O., Vanlandewijck Y.C., Manor G.L., Van Der Woude U.H.V.: A systematic review of wheelchair skills tests for manual wheelchair users with a spinal cord injury: towards a standardized outcome measure. Clinical Rehabilitation 24, 867-886 (2010)

3. Barea R., Boquete L., Rodríguez-Ascariz J.M., Ortega S., López E.: Sensory system for implementing a human-computer interface based on Electrooculography. Sensors Magazine 11, 310-328 (2011)

4. Perez C.: Real-time template based face and iris detection on rotated faces. International Journal of Opto-mechatronics 3, 54-67 (2009)

5. Mean-Foong O.: Hand gesture recognition: Sign to voice system (S2V). International Journal of Electrical and Electronics Engineering 3, 4-10 (2009)

6. Ville R., Pekka-Henrik N., Jarmo V., Jukka L.: Capacitive facial movement detection for human-computer interaction to click by frowning and lifting eyebrows. Med. Biol. Eng. Comput. 48, 39-47 (2010) 
7. Po-Yi S., Po-Chuan L., Jhing-Fa W., Yuan-Ning L.: Robust several-speaker speech recognition with highly dependable online speaker adaptation and identification. Elsevier Journal of Network and Computer Applications. 34, 1459-1467 (2011)

8. Salem C., Zhai S.: An isometric tongue pointing device. In: Proceedings of the ACM SIGCHI Conference on Human factors in computing systems, pp. 22-27 (1997)

9. Lau C., O'Leary S.: Comparison of computer interface devices for persons with severe physical disabilities. American Journal of Occupational Therapy 47, 102-103 (1993)

10. Huo X., Wang J., and Ghovanloo M.: A magneto-inductive sensor based wireless tongue-computer interface. IEEE Trans Neural Syst. Rehabil. Eng. 6(5), 497-504 (2008)

11. Johnson A.N., Huo X., Ghovanloo M. and Shinohara M.: Dual-task motor performance with a tongue-operated assistive technology compared with hand operations. Journal of NeuroEngineering and Rehabilitation 9(1), 1-16 (2012)

12. Huo X., Ghovanloo M.: Evaluation of a wireless wearable tongue computer interface by individuals with high-level spinal cord injuries. Journal of Neural Engineering $7(2), 1-12(2010)$

13. Lund M. E., Christiensen H.V., Caltenco H.A., Lontis E.R., Bentsen B., Andreasen Struijk L.N.: Inductive Tongue Control of Powered Wheelchairs. In: 32nd Annual International Conference of the IEEE EMBS, pp. 3361-3364 (2010)

14. Coy A., Barker J.: An automatic speech recognition system based on the scene analysis account of auditory perception. Elsevier Speech Communication 49, 384401 (2007)

15. Kim J., Huo X., Ghovanloo M.: Wireless Control of Smartphones with Tongue Motion Using Tongue Drive Assistive Technology. In: Proc. Annual International Conference of the IEEE Engineering in Medicine and Biology Society, pp. 5250$5253(2010)$

16. Huo X, Ghovanloo M.: Using unconstrained tongue motion as an alternative control surface for wheeled mobility. IEEE Trans on Biomed Eng 56(6), 1719-1726 (2009)

17. Giuca MR, Pasini M, Nastasio S, D' Ercole S., Tripodi D.: Dental and periodontal complications of labial and tongue piercing. Journal of biological regulators and homeostatic agents 26(3), 553-600 (2012) 